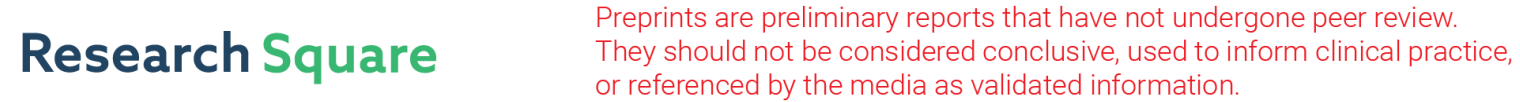 \\ Enhanced Magneto-Optical Effect in Three Layer Based Magnetoplasmonic Structures
}

Zeinab khaghani ( $\nabla$ khaghani.zeinab@yahoo.com )

University of Tabriz Faculty of Physics

Mahmood Hosseini Farzad

Shiraz University https://orcid.org/0000-0002-6356-0844

\section{Asghar Asgari}

University of Tabriz Faculty of Physics

\section{Research Article}

Keywords: surface plasmons (SP), magneto-optical effects, optical sensors, nanorods

Posted Date: February 15th, 2022

DOI: https://doi.org/10.21203/rs.3.rs-1272441/v1

License: (1) This work is licensed under a Creative Commons Attribution 4.0 International License.

Read Full License 


\title{
Enhanced Magneto-Optical Effect in Three Layer Based Magnetoplasmonic Structures
}

\section{Zeinab Khaghani ${ }^{\mathrm{a}}$, Mahmood Hosseini Farzad ${ }^{\mathrm{b} 1}$, Asghar Asgaric}

a-Faculty of Physics, University of Tabriz, Tabriz, Iran

b-Department of Physics, Shiraz University, Shiraz 71946-24795, Iran

c- Research Institute for Applied Physics and Astronomy, University of Tabriz, Tabriz, 51665-163, Iran

School of Electrical, Electronic and Computer Engineering, The University of Western Australia, Crawley, WA, 6009, Australia

Photonics Center of Excellence, University of Tabriz, Tabriz, 51666-14766, Iran

\begin{abstract}
In this article we introduce a magnetoplasmonics (MP) planar nanostructure with enhanced transverse magneto-optical (MO) activity. For this goal MO characteristics of magneto-structures comprising both metal layer and linear array of (metal) nanorods are analyzed. We explore three types of structures with nanorods and compare their features based on transvers magneto-optical Kerr effect (TMOKE) with the corresponding one for conventional three layers MO structure. Comparison between the values of TMOKE signals in two structures (proposed and conventional) shows more than one order of magnitude improvement. Due to this feature the refractive index sensitivity of the offered structures, specially with one and three types of Au nanorod arrays,
\end{abstract}

\footnotetext{
${ }^{1}$ corresponding author: hosseinif@shirazu.ac.ir
} 
appearance more than 2 order of magnitude larger than the conventional $\mathrm{Au} / \mathrm{Co} / \mathrm{Au}$ three layer $\mathrm{MO}$ structures. This notable response enhancement becomes serious affair in MP biosensing applications. We believe that our idea in these proposed nanostructures can be used in any other three layer based MO structures in the literature. In this basis, we apply this scenario to other types of such MO structures which leads to improvement in their MO activities and accordingly in their refractive index sensitivities.

Keywords:

surface plasmons (SP)

magneto-optical effects

optical sensors

nanorods

\section{Introduction}

Surface plasmon resonance (SPR) is an interesting effect which is utilized in advanced optical sensing [1]-[3]. This sensing mechanism have some positive features such as real-time detection, without fluorescent labeling identified chemical biological species with high resolution [4]. Nowadays, SPR sensors used in some important applications like double-stranded DNA recognition [5], pathogens sensing [6], and DNA sequences manifestation [7]. Propagated and localized surface plasmons occurred on flat plane and curved (nanoparticle) metal/dielectric interfaces, respectively, are two branches of SPR phenomenon [8], [9]. Collective motions of free electrons of metal at metal/dielectric interface which are excited by an TM electromagnetic wave, and coupled with it, produce a confined surface plasmon wave (SPW) propagating along the interface [8]. Different phase matching methods are exist for launching SPW's in several SPR 
device configurations such as prism coupling or attenuated-total-reflection (ATR), which is used in our analysis of this article, near field excitation by fiber tip [8] and gratting coupler [10].

Magneto-optical properties of a ferromagnetic metal film, like $\mathrm{Co}$ and $\mathrm{Fe}$, is improved when it lays adjacent to a noble metal film, due to SPR excitation on the noble metal and enhancement of electromagnetic field at its interface with ferromagnetic metal. On the other hand, due to the large absorption less of ferromagnetic metals the electromagnetic field of those excited SPW is rather weak and has a low sensitivity to the refractive index of surrounding medium. Therefore, the combination of two types of metals also compensate this sensing deficiency. Simple and the first magnetoplasmonic sensor like $\mathrm{Co} / \mathrm{Au}$ demonstrate more sensitivity compared to standard SPR sensor [11].

Here, we introduce a new three layer magnetoplasmonic nanostructure, by adjusting the individual thicknesses of each layer, and proved it possess more than one hundred-fold sensitivity increase over a standard MP sensor. Furthermore, we also expected this design leads to sensitivity enhancement in other three layer based MP heterostructures have been introduced in the literature until now. In the presence of a magnetic field, the dielectric tensor of the ferromagnetic material becomes non-diagonal [12]. The effect of an external magnetic field on the polarization state of the light transmitted and reflected by a material depends on the relative orientation of the magnetic field and the plane of incidence. When the magnetic field or the magnetization is set perpendicular to the plane of incidence of the light and parallel to the sample plane, known as the TMOKE, there is no change in polarization state. In this case, only the magnitude of the reflected light is affected by the magnetic field. In magnetoplasmonic (MP) structures, enhancement of MO activity is due to surface plasmon resonances [13]-[15]. Either in multilayer nanodiscs composed of $\mathrm{Au} / \mathrm{Co} / \mathrm{Au}$ or in $\mathrm{Au}$ were separated from continuous $\mathrm{Au} / \mathrm{Co} / \mathrm{Au}$ trilayers by a dielectric spacer shows how 
localized surface plasmon (LSP) excitation leads to an enhancement of the electromagnetic field in the MO active layer, which in turn causes improvement of the MO activity of the structure [16]. Plasmonic sensors are commonly used based on the concept of surface plasmon polaritons (SPPs) and in particular on the sensitivity of these resonances to the changes in the refractive index of the surrounding medium a metal structure [17].

It has been shown that the response of these sensors can enhance by using of MO properties of layered structures containing magnetic materials [11], [18]-[21]. Magneto-optical surface plasmon resonance (MOSPR) sensors have already been shown to have better properties than traditional SPRs ones [20], [22]- [26]. Improvement of gas sensing in magneto-optical SPR sensor by using and adding a nano-porous Tio2 layer on the $\mathrm{Au} / \mathrm{Co} / \mathrm{Au}$ multilayer is reported [27]. In the last decade, the TMOKE technique has been attracted the attention of researchers because of its potential in sensors [19], [27]-[35]. By measuring the TMOKE signal in $\mathrm{Au}-\mathrm{Co}-\mathrm{Au}$ films perforated with a periodic array of subwavelength holes, can leads to a large enhancement of the figure of merit of this type of hybrid MP crystal sensor [31]. In the $\mathrm{Au} / \mathrm{Co} / \mathrm{Au}$ trilayer structures, it has been shown that multi-layer magnetic structures with more than one magnetic layer increase the sensitivity of the sensor [23].

In this article, we introduced a new structure for observing the magnetoplasmonic effect to obtain an enhancement for transverse Kerr signal $\Delta R / R$ as a function of incident angle concerning the corresponding ones for other MP structures proposed before (up to our knowledge). For this goal, we introduce a structure composed of an array of Au nanorods lays on the Co layer and both are over a thin Au layer. Light is coupled to this structure from the Au layer side by using the usual Kretschmann configuration, thus a prism is attached to this side. One of the important results obtained from our simulation analysis belongs to the sharp variation of the transverse MO effect 
$\Delta \mathrm{R} / \mathrm{R}$, concerning light incident angle, around the plasmon resonance angle, wherein we have a minimum reflectance from the structure. The amplitude of this sharp rising and lowering in $\Delta R / R$ is large compared to corresponding values for other MP structures, at least one order of magnitude is improved. This comparison is pronounced specially for $\mathrm{Au} / \mathrm{Co} / \mathrm{Au}$ trilayers MP structure, work with surface plasmon polaritons, and for metal nanoparticles near the trilayer MP structure. Also, the refractive index sensitivity of our proposed structure is improved concerning the ordinary $\mathrm{Au} / \mathrm{Co} / \mathrm{Au}$ trilayers MP structure. This comparison is pronounced specially for three layer based MO structures (like $\mathrm{Au} / \mathrm{Co} / \mathrm{Au}$ ) such as $\mathrm{Cr} / \mathrm{Au} / \mathrm{Fe} / \mathrm{Au}$ and $\mathrm{Cr} / \mathrm{Co} / \mathrm{Cr} / \mathrm{Au}$, both of them are also studied in this article. Also, the refractive index sensitivity of such modified structures, according to the proposed idea which is applied to $\mathrm{Au} / \mathrm{Co} / \mathrm{Au}$, demonstrates an eligible improvement compared to the corresponding sensitivity of each structure in the conventional reported geometries (without metal nanorods).

\section{MO parameter and considered structures}

In our study, we use the TMOKE signal in which the sample magnetization is located on the sample plane and is perpendicular to the plane of incidence. In this category, the permittivity of the magnetic layer includes off-diagonal components related to the Voigt vector Q as follow [36]:

$$
\vec{\varepsilon}=\varepsilon\left(\begin{array}{ccc} 
& \cdot & \mathrm{iQy} \\
\cdot & 1 & \cdot \\
-i Q_{y} & \cdot & 1
\end{array}\right)
$$


$\mathrm{Q}_{\mathrm{y}}$ represents the MO constant of the material. The amplitude of the Voigt vector is taken to be considered as $\mathrm{Q}_{\mathrm{y}}=0.055$ - i0.013 [37]. The diagonal components $(\varepsilon)$ of the Co permittivity tensor were computed by the dielectric function data of materials (P. B. Johnson and R. W. Christy). In our study, the MO feature of the proposed structure is according to the calculation of reflectivity, $\mathrm{R}$, versus incident angle for a light with a wavelength of $653 \mathrm{~nm}$. Therefore the transverse Kerr signal $\Delta R / R$ versus incident angle is computed from its conventional definition as [36]:

$\Delta R / R=\frac{R_{p p}(+H)-R_{p p}(-H)}{R_{p p}(+H)+R_{p p}(-H)}$

Where $\mathrm{R}_{\mathrm{pp}}(+\mathrm{H})$ and $\mathrm{R}_{\mathrm{pp}}(-\mathrm{H})$ are the reflectance values for opposite magnetization directions. A schematic diagram of our structure under the Kretschmann excitation method is presented in Fig. $1(\mathrm{a})$. 


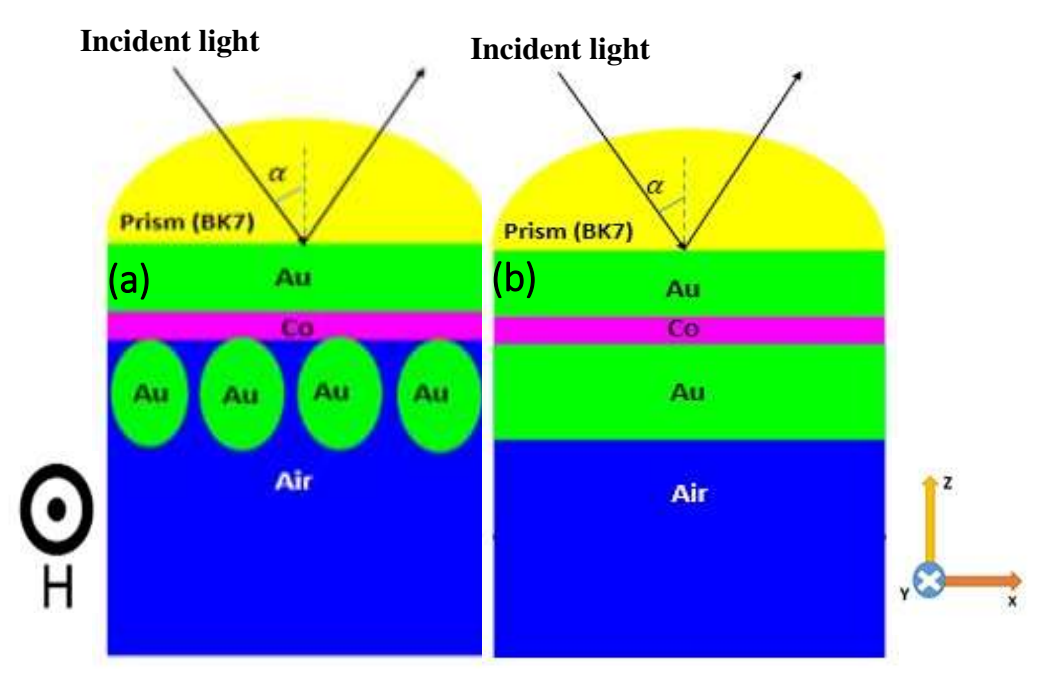

Fig. 1.a) Schematic diagram for our proposed structure consists of Au layer (16nm), magnetic material $\mathrm{Co}(4.5 \mathrm{~nm})$, an array of Au layer nanorods (Au NRs), or in a brief symbol $\mathrm{Au}(16 \mathrm{~nm}) / \mathrm{Co}(4.5 \mathrm{~nm}) / \mathrm{Au}$ NRs. The radius of each rod and the separation between them are $13 \mathrm{~nm}$ and $34 \mathrm{~nm}$, respectively. This structure is launched by prism coupling (BK7) from above. b) Picture of a conventional magnetoplasmonic structure $\mathrm{Au}(16 \mathrm{~nm}) / \mathrm{Co}(4.5 \mathrm{~nm}) / \mathrm{Au}(2 \times 13) \mathrm{nm}$, with the same thickness as our structure.

To investigate the magneto-optical properties theoretically, we consider an Au layer with 16nm thickness over a $4.5 \mathrm{~nm}$ thin layer of cobalt which are in turn stay on a one-dimensional array of Au nanorods. The radius of each rod is equal to $13 \mathrm{~nm}$ and the separation between them is $8 \mathrm{~nm}$ (center to center). All this structure is coated over a cylindrical BK7 prism, see Fig. 1(a). The light is coupled to this nanostructure from the prism side and the magnetic field is applied along the ydirection. For investigating the magnetooptical properties of our proposed structure we examine different structures, like panel (a) in Fig. 1, with different values of rod radii (and distance between rods) to obtain the best $\mathrm{MO}$ response among them. According to this task, we consider the structure 
$\mathrm{Au}(16 \mathrm{~nm}) / \mathrm{Co}(4.5 \mathrm{~nm}) / \mathrm{Au}$ NRs. The second panel in Fig. (1) shows the schematic representation of the conventional trilayer MO structure with the same geometry of our structure, panel (a), to compare the MO properties of two structures with each other. The other two appropriate structures are also considered in this article with three and two types of nanorods, see panels (a) and (b) in Fig. 2, respectively, to obtain a suitable MO response.

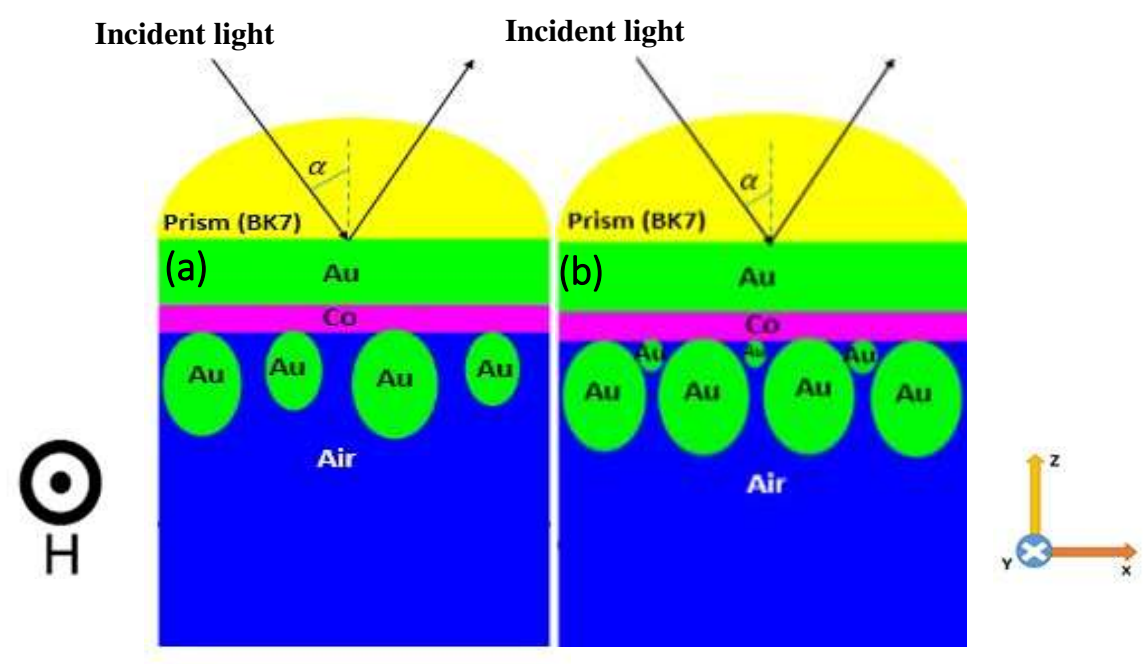

Fig. 2. Two other proposed structures with two (a) and three (b) types of nanorods. The thickness of each layer is like in Fig. 1(a) and smaller nanorods are the half size of larger ones $(6.5 \mathrm{~nm})$ in panel (a). two kinds of smaller nanorods between larger ones (13nm) in panel (b) have radii of $3 \mathrm{~nm}$ and $1.5 \mathrm{~nm}$.

\section{Result and discussion}

According to Fig. 1(a) we calculate by simulation the reflectivity from the proposed structure as a function of incident angle for a specific laser wavelength of $653 \mathrm{~nm}$. The results of this study for two directions of magnetization or magnetic field $(\mathrm{H})$ are plotted in Fig. 3(a). The same 
investigation is also carried out for the ordinary trilayer structure, Fig. 1(a), and the results are demonstrated in Fig. 3(b). As it is shown from Fig.3 the difference between reflectivity values for each direction of the $\mathrm{H}$ is very small, see for example the inset in panel (b). The reflectivity behavior of our MO structure has the main difference concerning the corresponding one for the ordinary MO structure. The increasing of the angular spread of the reflection minimum by an order of magnitude is this clear distinction. Furthermore, owing to this broadening the minimum value of reflectivity occurred at a larger light incident angle, see Fig. 3(a).
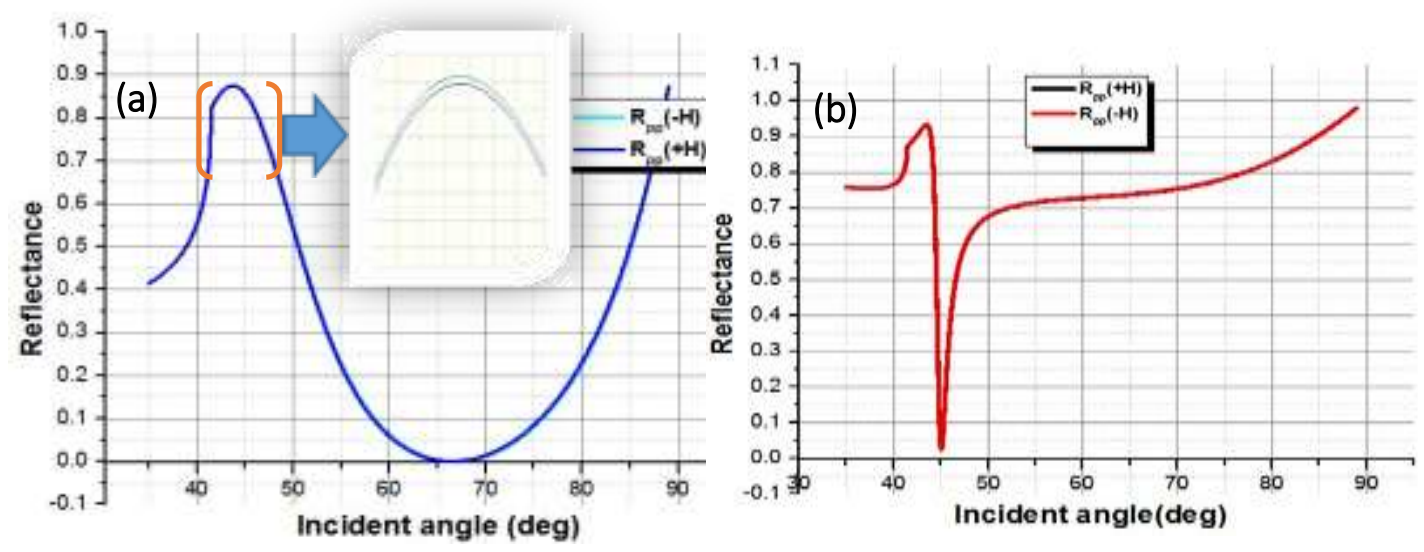

Fig. 3. Reflectivity as a function of incident angle for opposite directions of the external magnetic field, a) for proposed structure introduced in Fig. 1(a), (b) for conventional MP structure represented in Fig. 1(b).

On the other hand, from a plasmonic point of view, occurring the minimum with wide angular distribution represents the excitation of surface plasmon (SP) with higher confinement and larger dissipation in our structure concerning ordinary MO structure. This feature is confirmed by comparing the electric field (its magnitude) spatial distribution across the two structures at the angle of minimum reflectivity in each MO configuration, see two panels in Fig. 4. 


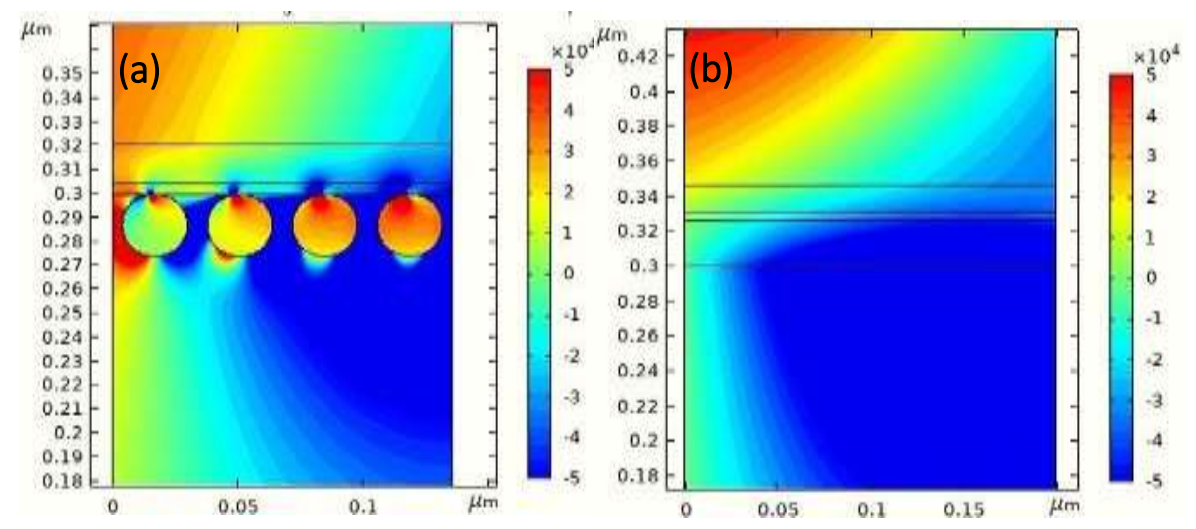

Fig. 4. Electric field (its amplitude) distributions at the cross-section of the offered structure with one type of nanorods (a) and for conventional trilayer MP structure (b), at the angle of minimum reflectivity.

As it is shown from panel (b) of this Figure more energy of the SPP mode is inside the homogenous surrounding dielectric environment (here is air) in ordinary MO structure. Contrary to this behavior is shown in a structure containing the rods and its penetration to the surrounding medium (air) is very low, see Fig. 4(a). Therefore, due to this important feature that appeared in our offered structure, we expect the improvement of coupling between surface plasmon resonances with magnetic layer $(\mathrm{Co})$ and accordingly enhancement of its $\mathrm{MO}$ characteristic. We are now in a position that to introduce the analysis of the transverse magneto-optical Kerr signal (TMOKE), equation (2), for structures depicted in Fig.1. The variations of $\frac{\Delta R}{R}$ for incident angles for structure panels (a) and (b) of this figure are indicated by the same panel names in Fig. (5). The TMOKE signal of our structure, panel (a), has a sharp variation around the minimum reflectivity angle. The same behavior is also shown for ordinary trilayer structure around its minimum reflectivity angle, see Fig. 5(b). The most specification of the former is a large increase in its maximum and minimum 
values of MO signal concerning corresponding values in the latter. Nearly, we experienced more than one order of magnitude improvement in MO signal.
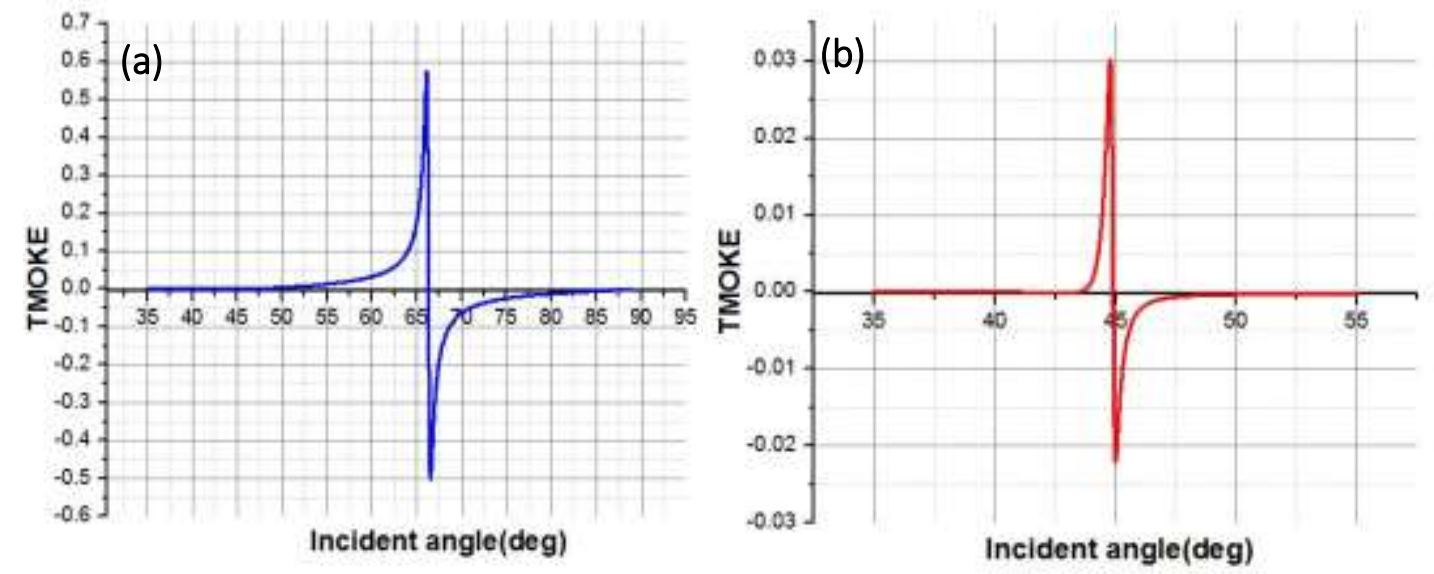

Fig. 5. TMOKE curve versus incident angle for a) $\mathrm{Au}(16 \mathrm{~nm}) / \mathrm{Co}(4.5 \mathrm{~nm}) / \mathrm{Au}$ NRs with one type of nanorods and b) ordinary MO structure $\mathrm{Au}(16 \mathrm{~nm}) / \mathrm{Co}(4.5 \mathrm{~nm}) / \mathrm{Au}(2 \times 13) \mathrm{nm}$.

Here we also examine the MO properties of the other two structures introduced in Fig. 2 by the same procedure discussed above. In panel (a) of this figure two kinds of nanorods are utilized despite the first proposed structure, Fig. 1(a), in which only one type of nanorod is employed. Smaller nanorods, with the half radius of adjacent rods, are between larger nanorods with the same lattice constant, 34nm. Fig. 2(b) demonstrates a MO structure with three types of metal nanorods such that between larger nanorods two models of smaller ones are situated, with radii of $3 \mathrm{~nm}$ and1.5 nm. The light reflectivity from these MO structures for opposite directions of magnetic fields is computed in Fig. 6. According to this figure, the shape of the reflectance diagram for structure with three types of nanorods has symmetry around its minimum, like the corresponding curve for the first proposed structure, see figure 3(a). 
There is no such symmetry for the reflectance curve in the two types of nanorod MO structure, see Fig. 6(a). On the other hand, the width of the valley around the minimum reflectance in this figure is smaller than the corresponding one for the three types of nanorods structure, see Fig. 6(b). The physical reason for this difference can be understood when the electric field distribution across the two structures at the angle of minimum reflectance are compared to each other, look at the two panels in Fig. 7.
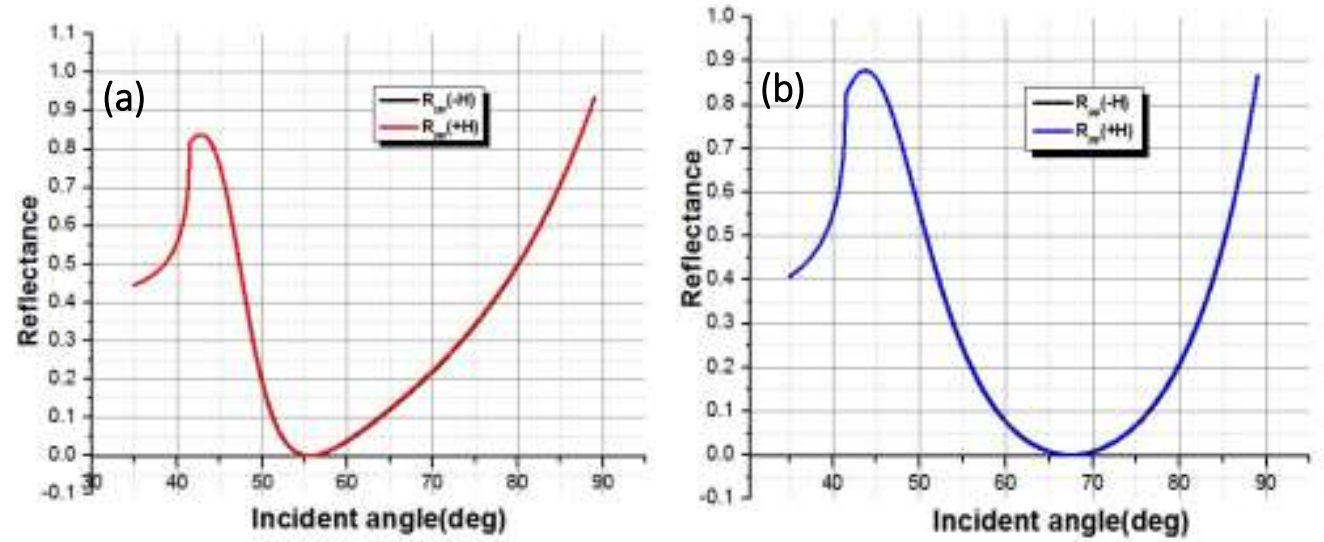

Fig. 6. Calculated of reflectivity versus incident angle structure in the presence of SPs excitation for a) $\mathrm{Au}(16 \mathrm{~nm}) / \mathrm{Co}(4.5 \mathrm{~nm}) / \mathrm{Au}$ NRs with two types of nanorods and b) $\mathrm{Au}(16 \mathrm{~nm}) / \mathrm{Co}(4.5 \mathrm{~nm}) / \mathrm{Au}$ NRs with three types of nanorods.
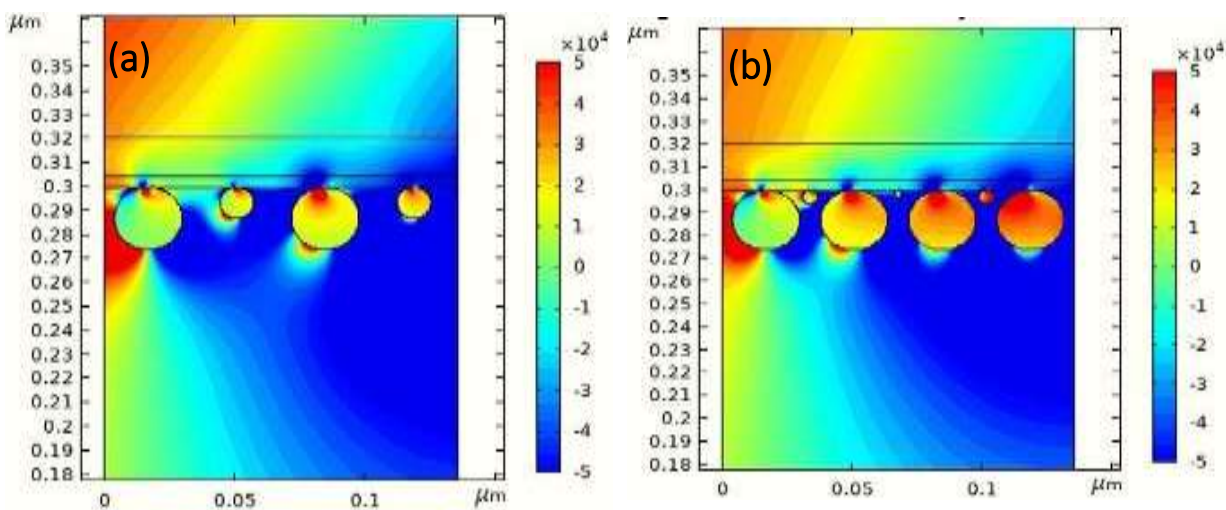

Fig. 7. Calculated electric field distributions for a) $\mathrm{Au}(16 \mathrm{~nm}) / \mathrm{Co}(4.5 \mathrm{~nm}) / \mathrm{Au}$ NRs with two types of nanorods at $55.08 \mathrm{deg}$ and b) $\mathrm{Au}(16 \mathrm{~nm}) / \mathrm{Co}(4.5 \mathrm{~nm}) / \mathrm{Au}$ NRs with three types of nanorods at $66.78 \mathrm{deg}$. 
Following the different colors around the nanorods in these figures one to be able to find that the field confinement to the metal nanorods is slightly increased in three types nanorods structure, panel (b), concerning other structure, panel (a).

In the other words, due to the shape of reflectance around its minimum in Fig. 6 (b) more confined surface plasmon modes are launched in the structure of Fig. 2(b) concerning corresponding modes in the structure of Fig. 2(a). After this physical inspection of the reflectivity properties of the two proposed structures introduced in Fig. (2) here we investigate their MO features. Inside the TMOKE signal of these conformations we observe the sharp high values of maximum and minimum adjacent to the corresponding angle of minimum reflectance, see two parts of Fig. (8). The same characteristic we also show before in the TMOKE signal for the first proposed structure with a single type nanorod, see Fig. 5(a).
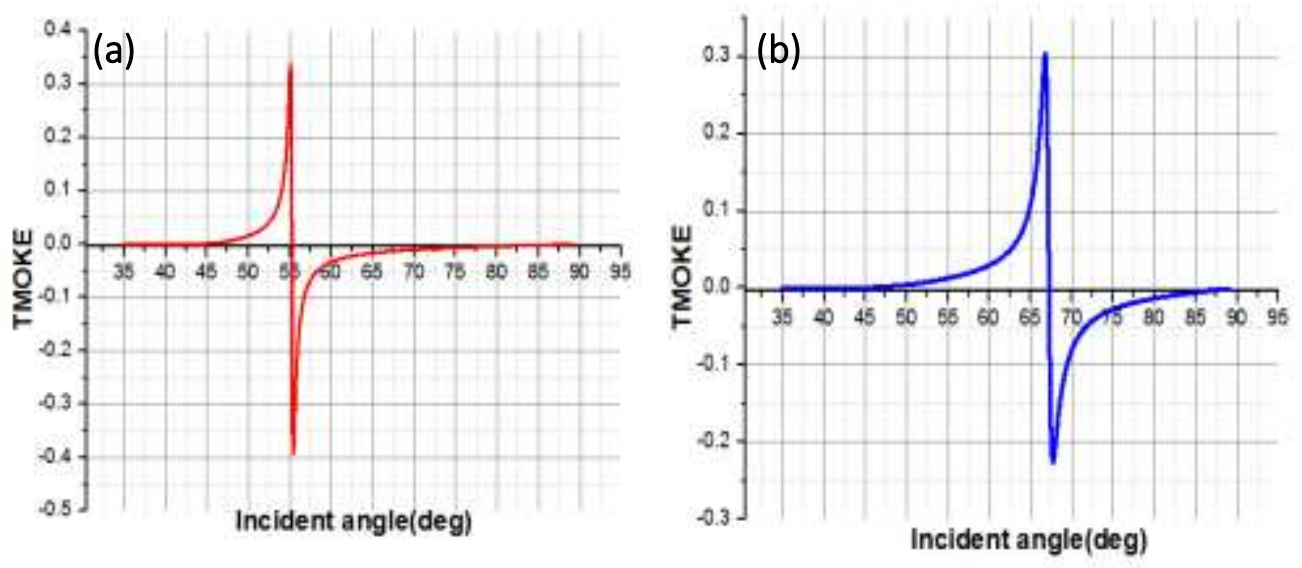

Fig. 8. TMOKE curve versus incident angle for a) $\mathrm{Au}(16 \mathrm{~nm}) / \mathrm{Co}(4.5 \mathrm{~nm}) / \mathrm{Au}$ NRs with two types of nanorods and b) $\mathrm{Au}(16 \mathrm{~nm}) / \mathrm{Co}(4.5 \mathrm{~nm}) / \mathrm{Au}$ NRs with three types of nanorods. 
The main difference between TMOKE signals of these structures compared to the corresponding signal of the first configuration (Fig. 4) is that in the latter the value of maximum (minimum) is nearly two times the same quantity values in the former.

The final study which is in accord with our motivation in the present task is about the sensing properties of the proposed structures. For this goal considering the refractive index of the surrounding medium of the nanorods experienced a little change concerning the vacuum index $(n=1)$.

According to Fig. 9, one can show that our proposed structure with single-type nanorods has exceeded refractive index sensitivity for ordinary trilayer structure, assimilate panel (a) with panel (b). The separation between TMOKE signal curves for successive little variation of refractive index, $\Delta \mathrm{n}=0.002$, is more pronounced in the proposed MO structure. Furthermore, the magnitude of the maximum (minimum) in each TMOKE curve is also increased by augmenting the refractive index by a little value in each step. This feature is not remarked in ordinary MO structure, see Fig. 9(b). 

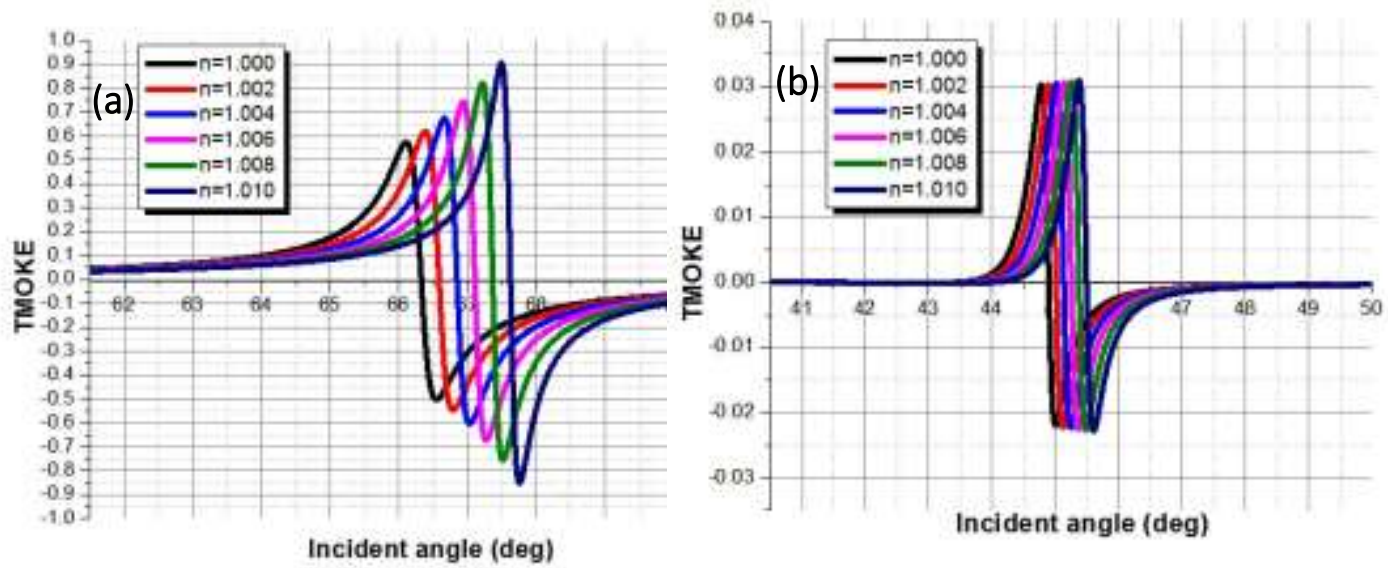

Fig. 9. a) TMOKE signal as a function of the incident angle at the wavelength of $653 \mathrm{~nm}$ for the different refractive indexes of the surrounding medium. a) for the proposed structure with one type of nanorods, b) for conventional trilayer MO structure, see Figures 1(a) and 1(b), respectively.

The same investigation we also carried out for other proposed MO structures with two types of nanorods, regarding panel (a) of Fig.10, and three kinds of nanorods, considering panel (b) of this figure. The general behavior of TMOKE curves for these structures, Fig. (10), is the same as the corresponding one for the first proposed structure, look Fig. 9(a), but with a moderate enhancement characteristic. Here, we use the standard criterion for evaluating the sensitivity enhancement of these proposed MO structures. 

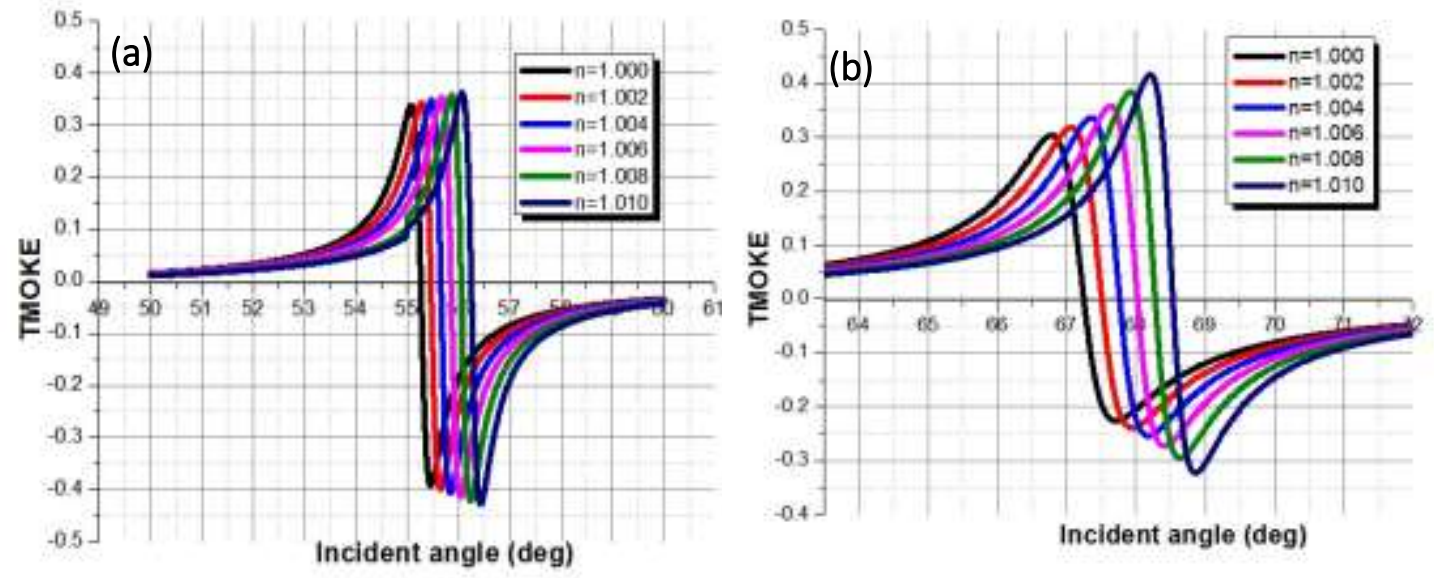

Fig. 10. a) TMOKE signal as a function of the incident angle at the wavelength of $653 \mathrm{~nm}$ for the different refractive indexes of the surrounding medium. a) for the proposed structure with two types of nanorods, b) for the offered structure with three types of nanorods, see Figures 2(a) and 2(b), respectively.

Theoretical analysis of the response of a magnetoplasmonic TMOKE sensor or its sensitivity can be defined as [30]:

$\eta=\frac{\partial S}{\partial n_{e n}}=\frac{\partial S}{\partial \theta} \times \frac{\partial \theta_{\max }}{\partial n_{e n}}$

Here, $\mathrm{S}$ represents the maximum value of the TMOKE signal that occurred at a certain angle $\left(\theta_{\max }\right)$ for each $n_{\text {en, }}$ where $n_{\text {en }}$ is the refractive index of the medium around the nanorods. Then from Eq (3) one can find that the two factors contribute to expected sensitivity (1), the slope of the TMOKE curve as a function of the incident angle $(\theta)$ and the angular displacement of such curve 
when nen changes. We extract the slope of two lines partitioning to these factors from all TMOKE curves appear in figures (9) and (10) for the whole contemplated MO structures.

For example, the variation of the maximum TMOKE signal concerning its occurrence angle $\left(\theta_{\max }\right)$, extracted from Fig. 9(a), is represented in Fig. 11(a). panel (b) of this figure also produce from Fig. 9 (a) and shows that the line belongs to alteration of $\theta_{\max }$ versus a change of refractive index.
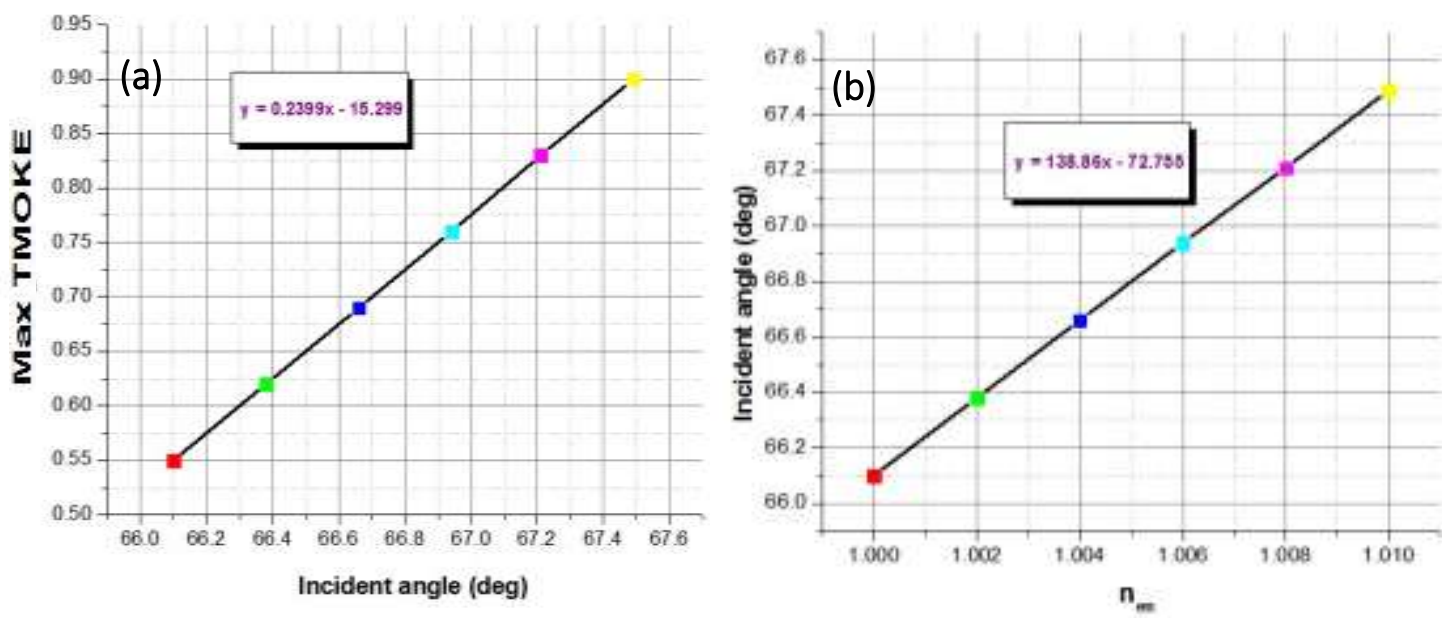

Fig. 11. a) Linear relation between maximum TMOKE signal versus its occurrence angle, whenever the surrounding refractive index of the MO structure with single kind of nanorods is changed. b) the change of angle, in which the TMOKE signal becomes maximum, versus the variation of the refractive index. 

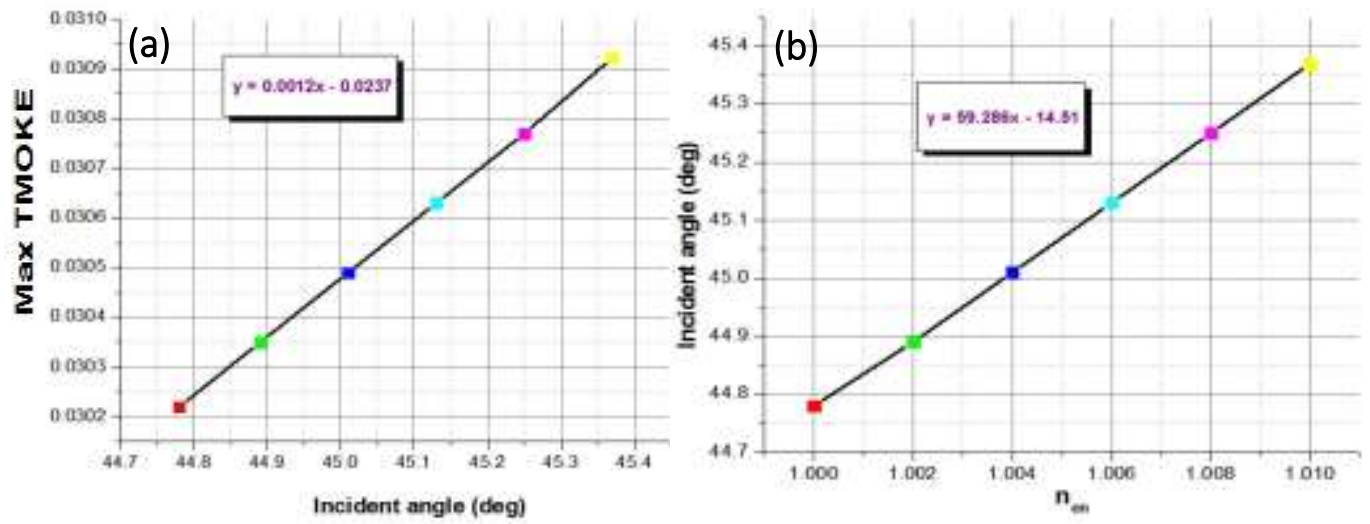

Fig. 12. a) Like the same descriptions of the panels (a) and (b) in the caption of Figure (11), but for trilayer conventional MO structure.
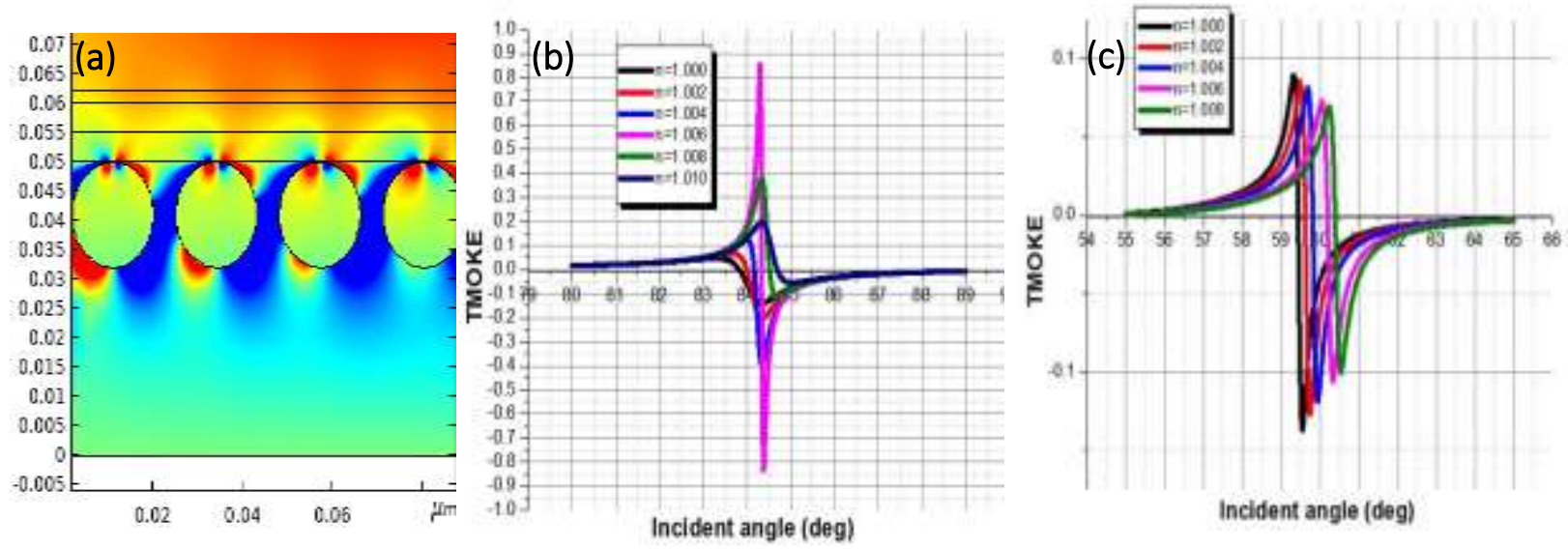

13. a) Calculated electric field (magnitude) distribution for modified structure contain one type of nanorods, $\mathrm{Cr}(2 \mathrm{~nm}) / \mathrm{Au}(5 \mathrm{~nm}) / \mathrm{Fe}(5 \mathrm{~nm}) / \mathrm{Au} \mathrm{NRs}$, (radius of each rod and the separation between them are $9 \mathrm{~nm}$ and $23 \mathrm{~nm}$, respectively) at the angle that its TMOKE is maximum for air surrounding medium $(n=1.000)$. b) TMOKE diagrams of this structure for different refractive indices (with a very small differential value between those $\Delta \mathrm{n}=0.002$ ) of the surrounding medium. c) The corresponding TMOKEc urves for the original structure, before replacing narorods instead of $\mathrm{Au}$ layer [19]. 

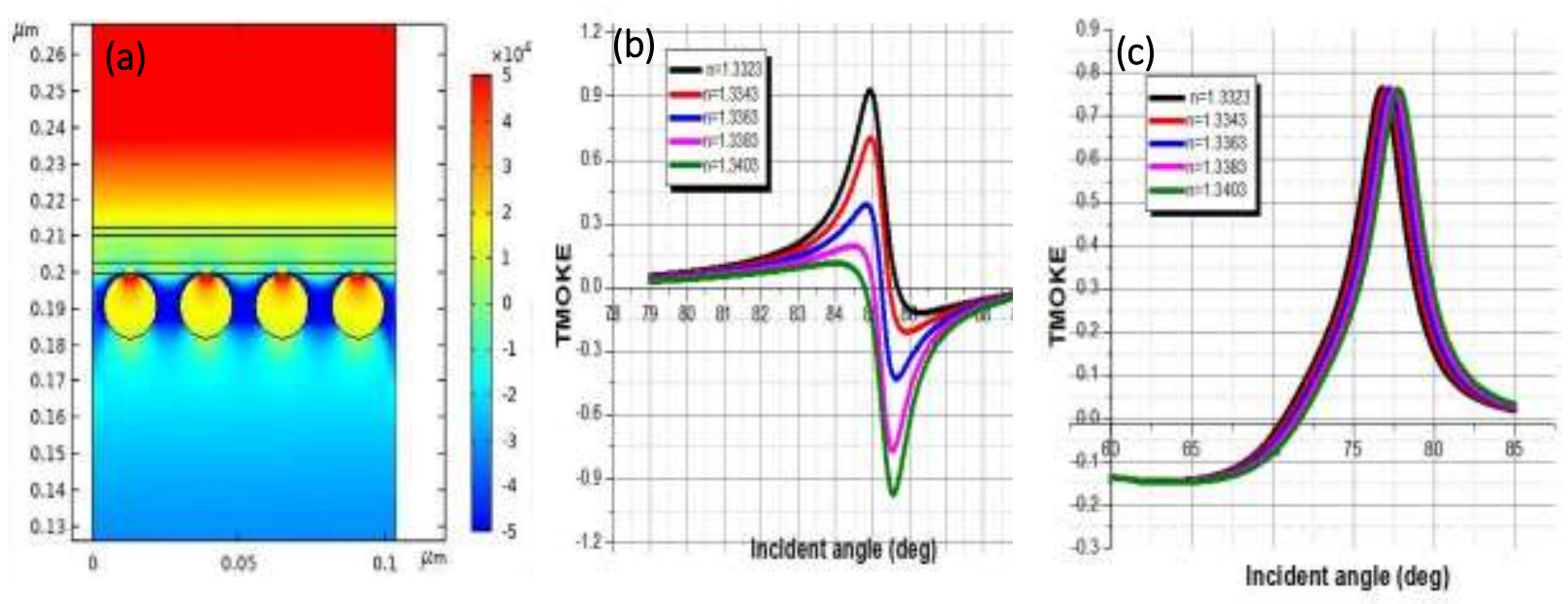

Fig. 14. a) Calculated electric field distribution for structure contains nanorods (radius of each rod and the separation between them are $9 \mathrm{~nm}$ and $26 \mathrm{~nm}$, respectively), $\mathrm{Cr}(2 \mathrm{~nm}) / \mathrm{Co}(7.5 \mathrm{~nm}) / \mathrm{Cr}(3 \mathrm{~nm}) / \mathrm{Au} \mathrm{NRs}$, at $84.94 \mathrm{deg}$ in which the maximum TMOKE is occurred for $n=1.3323$. b) Variation of TMOKE signal versus incident angle of this modified structure when the refractive indices of the surrounding medium change very small. c) The corresponding TMOKE diagrams for the original layered structure recorded in the literature [11].

Hence from Fig. (11) the sensitivity of the proposed MO structure with a single type of nanorods is obtained equal to 33.313. After applying the same scenario for other concerned MO structures, we computed the following sensitivity values for structures with conventional trilayer see Fig. (12), two and three types of nanorods are $0.0711,2.491$, and 11.847 respectively. Therefore, based on these results proposed MO structures with one, two, and three types of nanorods increased the refractive index sensitivity by the factors of $468.5,35$, and 166.6 , respectively, when compared to the same parameter for conventional trilayer MO structure, production from Fig. (12). Among these structures, the sensitivity enhancement of the first one is more pronounced, and it will be helpful in many exhaustive sensing applications. 
In order to confirm our idea, as discussed above for $\mathrm{Au} / \mathrm{Co} / \mathrm{Au}$ three layer structure, have a positive effect on MO activity of other three layer based structures we exam this scenario for two three layers famous MO structures. As it is shown from Figs. 13(a) and (b) we represent an electric field distribution and TMOKE characteristic of the modified multilayer configuration, its conventional geometry which is reported in reference [19] is in the form of $\mathrm{Cr} / \mathrm{Au} / \mathrm{Fe} / \mathrm{Au}$. Here, only the last $\mathrm{Au}(30 \mathrm{~nm})$ layer is replaced by a designed single array of $\mathrm{Au}$ nanorods. The improvement of the TMOKE signal is emerged after this modification and it is understand by comparing the panels (b) and (c) of Fig. 13. By calculating the refractive index sensitivity deduced from these figures one can obtain the values of 48.28 and 2.73 for modified and original structures, panels (b) and (c), respectively, and the enhancement factor of 17.7. The same procedure we act on the other conventional MO structure in the literature [11] in order to check the improvement model worked very well. The obtained results for this action is demonstrated in different panels of Fig .14. Panel (a) shows the modified structure of the reported one, $\mathrm{Cr} / \mathrm{Co} / \mathrm{Cr} / \mathrm{Au}$ layers, and part (b) is the TMOKE signal of this structure for different refractive index of the surrounding medium of the nanorods.

The small variation of the refractive index (around $n=1.3363$ ) produces an effective change in the TMOKE diagrams of the modified structure, see panel (b). However, this behavior is not shown in the TMOKE signal of the original three layer structure, $\mathrm{Cr} / \mathrm{Co} / \mathrm{Cr} / \mathrm{Au}$ layers, see panel (c) of Fig. 14. It is interesting to calculate the sensitivity, according to Eq. (3) and its discussion, by using of these two TMOKE diagrams. The results obtain from these computations manifest an improvement factor of 224.6 in the refractive index sensitivity of the modified structure. It is a remarkable enhancement, more than two order of magnitude, in sensitivity of the structures with MO activity. 


\section{4. conclusion}

Here, we show that the magnetoplasmonic performance of nanostructures that contain both noble metal thin layer and nanorods, with a suitable diameter and lattice constant, is better than the corresponding one for conventional MP structure with two metal layers around the thin magnetic material. This comparison is based on simulation of their TMOKE signals as a function of light incident angle. The maximum (minimum) value of the TMOKE signal evince more than one order of magnitude growth in the proposed structure with one type of nanorods. Also, improvement of more than two order of magnitude in refractive index response of the offered structures, with one and three types of nanorods, recommend these magnetoplasmonic design structures for more competent biosensing applications in the progressive magneto-optic field. Finally, it is predicted that by using of this idea in other three (conventional) layer based magnetoplasmonic structures with an adjusting design (for nanorods) leads to improvement of their magneto-optic features. We apply this scenario to different layered MO structurers reported in literature in order to proved this expectation and one of them shows a remarkable enhancement, more than 2 order of magnitude, in its sensitivity feature.

\section{References}

[1] C. Republic, S. Surface, and R. S. Coverage, Surface Plasmon Resonance Sensors for Detection of Chemical and Biological Species, 462-49(2008)

[2] B. D. Gupta, S. K. Srivastava, R. Verma, Fiber Optic Sensors Based on Plasmonics, World Scientific, (2015)

[3] J. Homola, Surface plasmon resonance based sensors, Springer Science \& Business Media. 4, (2006) 
[4] S.Szunerits, R. Boukherroub. Introduction to plasmonics: Advances and applications, (2015)

[5] O. Slinchenko, A. Rachkov, H. Miyachi, M. Ogiso, and N. Minoura, Imprinted polymer layer for recognizing double-stranded DNA, Biosensors and Bioelectronics. 20, 1091-1097(2004)

[6] B. Oh, Y. Kim, W. Lee, Y. Min, W. Hong, and J. Choi, Immunosensor for detection of Legionella pneumophila using surface plasmon resonance, Biosensors and Bioelectronics. 18, 605-611(2003)

[7] R. Wang, M. Minunni, S. Tombelli, and M. Mascini, A new approach for the detection of DNA sequences in amplified nucleic acids by a surface plasmon resonance biosensor, Biosensors and Bioelectronics. 20, 598605. (2004)

[8] S. A. Maier, Surface plasmon polaritons at metal/insulator interfaces, in Plasmonics: Fundamentals and Applications, Springer 21-37 (2007)

[9] V. Klimov, Nanoplasmonics. CRC press, (2014)

[10] B. K. Singh and A. C. Hillier, Surface Plasmon Resonance Enhanced Transmission of Light through Gold Coated Diffraction Gratings Surface Plasmon Resonance Enhanced Transmission of Light through, Analytical Chemistry. 80, 3803-3810 (2008)

[11] B. Sepúlveda, A. Calle, L. M. Lechuga, and G. Armelles, Highly sensitive detection of biomolecules with the magneto-optic surface-plasmon-resonance sensor, Opt. Lett. 31 1085-1087 (2006)

[12] A. K. Zvezdin and V. A. Kotov, Modern magnetooptics and magnetooptical materials. CRC Press, (1997)

[13] V. I. Safarov, V. A. Kosobukin, C. Hermann, G. Lampel, J. Peretti, and C. Marlière, Magneto-optical effects enhanced by surface plasmons in metallic multilayer films, Phys. Rev. Lett. 73 3584-3587 (1994)

[14] C. Hermann, V. A. Kosobukin, G. Lampel, J. Peretti, V. I. Safarov, and P. Bertrand, Surface-enhanced magneto-optics in metallic multilayer films, Phys. Rev. B. 64235422 (2001) 
[15] E. Ferreiro-Vila et al., Surface plasmon resonance effects in the magneto-optical activity of $\mathrm{Ag}-\mathrm{Co}-\mathrm{Ag}$ trilayers, IEEE Trans. Magn. 44 3303-3306 (2008)

[16] G. Armelles et al., Magnetoplasmonic nanostructures: systems supporting both plasmonic and magnetic properties, J. Opt. A Pure Appl. Opt. 11114023 (2009)

[17] S. Afsheen, A. Ahmad, T. Iqbal, M. Ijaz, and A. Bashir, Optimizing the Sensing Efficiency of Plasmonic Based Gas Sensor, Plasmonics. 16 541-546 (2021)

[18] J. B. González-Díaz et al., Surface-magnetoplasmon nonreciprocity effects in noble-metal/ferromagnetic heterostructures, Phys. Rev. B.76 153402 (2007)

[19] D. Regatos et al., Au/Fe/Au multilayer transducers for magneto-optic surface plasmon resonance sensing, J. Appl. Phys. 108 (2010)

[20] D. Regatos, B. Sepúlveda, D. Fariña, L. G. Carrascosa, and L. M. Lechuga, Suitable combination of noble/ferromagnetic metal multilayers for enhanced magneto-plasmonic biosensing, Opt. Express. 19 8336$8346(2011)$

[21] M. G. Manera, E. Ferreiro-Vila, J. M. Garcia-Martin, A. Garcia-Martin, and R. Rella, Enhanced antibody recognition with a magneto-optic surface plasmon resonance (MO-SPR) sensor, Biosensors and Bioelectronics. 58114-120 (2014)

[22] C. Clavero, K. Yang, J. R. Skuza, and R. A. Lukaszew, Magnetic field modulation of intense surface plasmon polaritons, Opt. Express. 18 7743-7752 (2010)

[23] K. Kämpf, S. Kübler, F. Wilhelm Herberg, and A. Ehresmann, Magneto-optic surface plasmon resonance optimum layers: Simulations for biological relevant refractive index changes, J. Appl. Phys. 112, (2012)

[24] C. Rizal, S. Pisana, and I. Hrvoic, Improved magneto-optic surface plasmon resonance biosensors, Photonics. $5(2018)$ 
[25] C. Rizal, S. Pisana, I. Hrvoic, and E. E. Fullerton, Microstructure and magneto-optical surface plasmon resonance of Co/Au multilayers, J. Phys. Commun. 2, (2018)

[26] C. Rizal, V. Belotelov, D. Ignatyeva, A. K. Zvezdin, and S. Pisana, Surface plasmon resonance (SPR) to magneto-optic SPR, Condens. Matter. 4, 1-7 (2019)

[27] M. G. Manera et al., Enhanced gas sensing performance of TiO2 functionalized magneto-optical SPR sensors, J. Mater. Chem. 21, 16049-16056 (2011)

[28] M. G. Manera et al., Enhanced gas sensing performance of TiO 2 functionalized magneto-optical SPR sensors, J. Mater. Chem. 21, 16049-16056 (2011)

[29] M. G. Manera et al., TiO 2 brookite nanostructured thin layer on magneto-optical surface plasmon resonance transductor for gas sensing applications, J. Appl. Phys. 112,( 2012)

[30] G. Pellegrini and G. Mattei, "High-Performance Magneto-Optic Surface Plasmon Resonance Sensor Design: An Optimization Approach,” Plasmonics. 9, 1457-1462 (2014)

[31] B. Caballero, A. Garciá-Martín, and J. C. Cuevas, "Hybrid Magnetoplasmonic Crystals Boost the Performance of Nanohole Arrays as Plasmonic Sensors," ACS Photonics. 3, 203-208 (2016)

[32] A. A. Grunin, I. R. Mukha, A. V. Chetvertukhin, and A. A. Fedyanin, Refractive index sensor based on magnetoplasmonic crystals, J. Magn. Magn. Mater. 415, 72-76 ( 2016)

D. O. Ignatyeva, G. A. Knyazev, P. O. Kapralov, G. Dietler, S. K. Sekatskii, and V. I. Belotelov, Magnetooptical plasmonic heterostructure with ultranarrow resonance for sensing applications, Sci. Rep. 6, $1-7(2016)$

[34] N. Li, T. Tang, J. Li, L. Luo, P. Sun, and J. Yao, Highly sensitive sensors of fluid detection based on magnetooptical optical Tamm state, Sensors Actuators, B Chem. 265, 644-651 (2018)

[35] C. Rizal and V. Belotelov, Sensitivity comparison of surface plasmon resonance (SPR) and magneto-optic SPR biosensors, Eur. Phys. J. Plus. 134, (2019) 
[36] M. B. Ross, M. R. Bourgeois, C. A. Mirkin, and G. C. Schatz, Magneto-Optical Response of Cobalt Interacting with Plasmonic Nanoparticle Superlattices, J. Phys. Chem. Lett. 7, 4732-4738 (2016)

[37] R. Osgood, K. Riggs, A. E. Johnson, J. Mattson, C. Sowers, and S. Bader, Magneto-optic constants of hcp and fcc Co films, Phys. Rev. B - Condens. Matter Mater. Phys. 56, 2627-2634 (1997) 